\title{
Nanoscale Topographic and Biomechanical Studies of the Human Internal Limiting Membrane
}

\author{
Paul B. Henrich, ${ }^{1}$ Christophe A. Monnier, ${ }^{2}$ Willi Halfter, ${ }^{3}$ Christos Haritoglou, ${ }^{4}$ \\ Rupert W. Strauss, ${ }^{5,6}$ Roderick Y. H. Lim, ${ }^{2}$ and Marko Loparic ${ }^{2}$
}

Purpose. The purpose of this article was to create a nanometer scale topographic and biomechanical profile of the human internal limiting membrane (ILM) under native conditions.

Methods. ILMs from the posterior pole of postmortem human eyes were prepared as flat mounts and investigated by atomic force microscopy (AFM) under physiological conditions. Structural analysis was complemented by transmission electron microscopy.

Results. Average thickness of the fully hydrated, native ILMs was $3488 \pm 460 \mathrm{~nm}$. Thickness variations from $100 \mathrm{~nm}$ to $4326 \mathrm{~nm}$ characterized the fovea, which displayed a craterlike morphology. Outside the fovea, thickness distribution was uniform. Although mean ILM thicknesses were similar, standard deviation was higher on the retinal than on the vitreal side, indicating greater roughness. Average ILM stiffness was more than fivefold higher on the retinal than on the vitreal side (227 vs. $44 \mathrm{kPa})$.

Conclusions. A detailed topographical and nanomechanical profile of native human ILM was generated using AFM. Thickness values were significantly higher than in previous studies because of the preservation of native conditions. Both thickness and stiffness showed marked variations around the fovea but were relatively uniform outside the foveal area. Interestingly, the foveal ILM displayed a craterlike morphological appearance with four distinct layers separated by comparatively steep thickness increments. ILM stiffness was considerably higher on the retinal than on the vitreal side. AFM opens new possibilities for investigating native basement membranes under physiological and pathological conditions. Transmission electron microscopy revealed higher extracellular matrix protein density on the retinal than on the vitreal side. (Invest Ophthalmol Vis Sci. 2012;53:2561-2570) DOI:10.1167/iovs.11-8502

From the ${ }^{1}$ University of Basel, University Hospital, Department of Ophthalmology, Basel, Switzerland; ${ }^{2}$ University of Basel, Biozentrum and the Swiss Nanoscience Institute, Basel, Switzerland; ${ }^{3}$ Department of Neurobiology, University of Pittsburgh, Pittsburgh, Pennsylvania; ${ }^{4}$ University Eye Hospital of the Ludwig-Maximilians University, Munich, Germany; ${ }^{5}$ Department of Ophthalmology and Optometry, Linz General Hospital, Linz, Austria; and ${ }^{6}$ Department of Ophthalmology, Medical University Graz, Graz, Austria.

Supported by a Swiss CTI (Commission for Technology and Innovation) Project (11977.2 PFNM-NM), entitled ARTIDIS (Automated and Reliable Tissue Diagnostics) (ML, RYHL).

Submitted for publication August 30, 2011; revised December 20, 2011 and February 6, 2012; accepted February 20, 2012.

Disclosure: P.B. Henrich, None; C.A. Monnier, None; w. Halfter, None; C. Haritoglou, None; R.W. Strauss, None; R.Y.H. Lim, CTI (F); M. Loparic, CTI (F)

Corresponding author: Paul B. Henrich, Department of Ophthalmology, University Hospital Basel, PO Box, CH-4012 Basel, Switzerland; Henrichp@uhbs.ch.

Investigative Ophthalmology \& Visual Science, May 2012, Vol. 53, No. 6

Copyright 2012 The Association for Research in Vision and Ophthalmology, Inc.
The epithelial/mesenchymal interface of most tissues is 1 marked by the presence of a basement membrane (BM). These thin, uniform insoluble sheets of highly specialized extracellular matrix (ECM) proteins are found in all multicellular organisms, ${ }^{1}$ but vary in composition in a tissue-specific manner. ${ }^{2}$ BMs serve as substrates for epithelial cells, endothelial cells, and myotubes. ${ }^{3}$ They also contribute to the mechanical strength of their neighboring tissues. ${ }^{4}$ The internal limiting membrane (ILM) is located at the vitreal border of the retina and is one of six BMs of the eye. ${ }^{3}$ Its molecular constituents include members of the laminin, nidogen, collagen IV, and proteoglycan families. ${ }^{2,5}$

Our current knowledge of topographic variations within the ILM is mostly based on light microscopy (LM) ${ }^{6}$ and transmission electron microscopy (TEM) studies $^{7,8}$ of dehydrated and fixated specimens. For example, for smaller mammalian species, such as the rabbit, TEM results revealed thin and uniformly smooth ILMs, ${ }^{9}$ whereas primates display much thicker ILMs with complex folds on their retinal side, matching the convoluted surface of the Müller cell endfeet. TEM studies of human ILM revealed conspicuous spatial variations in thickness ranging from $20 \mathrm{~nm}$ in the pit of the fovea, to an average of $2500 \mathrm{~nm}$ in the extrafoveal posterior region $^{8,9}$ and only $51 \mathrm{~nm}$ near the vitreous base. ${ }^{8}$ It is considered that because of highly irregular "crypts, valleys, and ridges" of the retinal surface, ${ }^{9}$ particularly near the posterior pole, the ILM exhibits a high degree of thickness variability. $^{8,9}$

A recent study has shown that ILM represents a typical BM in terms of ultrastructure and biochemical composition. ${ }^{4}$ The same study revealed that the mechanical strength of the ILM may be essential for normal eye size development. ${ }^{4}$

The ILM is of particular interest among both scientists and clinicians, as it is accessible for direct biochemical and biomechanical analysis. It also contains the site of vitreoretinal adhesion and traction, abnormalities which are considered to be fundamental for the development of numerous diseases, including epiretinal fibrosis, vitreoretinal traction syndrome, persistent macular edema, and macular hole formation. ${ }^{10}$ Vitreoretinal traction is most thoroughly addressed through surgical excision of the pathological ILM, ${ }^{11}$ as mechanical separation of the vitreous from the retina is often incomplete and vitreous fibrils may remain on the surface of the ILM. ${ }^{12}$

Because the ILM is translucent and delicate, its removal can be technically challenging, with the risk of retinal complications, including intraretinal hemorrhages, central retinal breaks, and functional retinal injury, ${ }^{13-15}$ as well as incomplete ILM removal. There have been numerous attempts to promote safe and complete ILM removal by optimizing vital dyes for ILM staining, ${ }^{16-18}$ the application of adjuvant pharmaceuticals, and repeated modifications of microsurgical instruments ${ }^{10}$ to improve surface grip.

Both ILM thickness and biomechanical characteristics influence peeling behavior; however, little is known on the 
intrinsic material properties of the native ILM. ${ }^{19}$ A previous study demonstrated an increase in tensile stiffness in a biomechanical tester after the exposure of strips of retina from postmortem porcine eyes to indocyanine green (ICG) and halogen light owing to a cross-linking effect. ${ }^{20}$ By means of bright field microscopy and TEM studies, an enhanced detachment of the ILM from the underlying retina was observed in glial fibrillary acidic protein and/or vimentin knockout mice (GFAP-/-, Vim-/- and GFAP-/-, Vim-/-) caused by an increased disruption of Müller cell processes proximal to their endfeet. ${ }^{21}$ Separately, these two studies focus on highly specific issues. A more general description of biomechanical behavior of the native vitreoretinal interface is not available.

To this end, we have used atomic force microscopy (AFM) as a unique tool to visualize, manipulate, and quantitatively assess structural and biomechanical characteristics of native biological samples at nanometer scale resolution. ${ }^{22,23}$ In this study, we show that AFM has the advantage of resolving the nanoscale anatomical and physiological properties of the fully hydrated native human ILM. In particular, we established a structural and nanomechanical profile of the vitreous and retinal side of the ILM. Our study focuses on the ILM close to the fovea, an area of high clinical interest and target of numerous ILM peeling procedures.

\section{Materials ANd Methods}

\section{Specimen Preparation}

The material used in this study consisted of postmortem adult human eyes, obtained from the Center of Organ Recovery and Education of the University of Pittsburgh. The use of human eyes was approved by the institutional review board (IRB) of the University of Pittsburgh under the IRB Protocol number 0312072.

ILMs from defined areas of human retina were isolated by treatment of retinal segments with Triton-X-100 and deoxycholate as described earlier. ${ }^{3}$ All retinal segments were from the posterior retinal pole from within the large temporal vascular arcades. The area within the vascular arcades was subdivided into a grid of 9 segments of approximately $2 \times 2$-mm side length each (Fig. 1) so as to allow spatial quantification of thickness and roughness observations. The central square was centrally placed on the fovea (Figs. 1A, 1B), and the outer margins of the nasal, superior, and inferior segments were located in close vicinity to the optic disc and the superior and inferior temporal vascular arcades, respectively. The central square was designated as "central quadrant," the surrounding squares as "midperiphery" or "midperipheral quadrants."

ILM flat-mount preparation was as described earlier. ${ }^{3}$ Briefly, ILM samples from each defined segment were transferred under PBS onto glass slides coated with $10 \mu \mathrm{g} / \mathrm{mL}$ Poly-lysine (P-1524; Sigma-Aldrich, St. Louis, MO). The central area of the slide was encircled with a PAP-Pen (Research Products International, Mt. Prospect, IL) to contain the PBS droplet around the ILM sample. Excessive PBS was removed and the slides were centrifuged at $1000 \mathrm{rpm}$ for 3 minutes to firmly attach the ILMs to the glass slides. The immobilized ILMs were kept under PBS for immediate use or stored in PBS in a $50-\mathrm{mL}$ tube at $4^{\circ} \mathrm{C}$. The samples were never frozen. Importantly, all AFM measurements were performed while the ILM was fully submerged in PBS.

ILM sets of 10 eyes from nine human donors were prepared. Donors were Caucasians, aged 52 to 83 years (mean 69.8 years). The fineness of the tissue allowed complete preservation of all quadrants in one eye only (sample 5). All other grids were partially incomplete because of the loss of some segments throughout the preparation process (Table 1). Because of the particularly thin ILM in the immediate foveal area, preparation of specimens of the central quadrant proved especially challenging. The complete foveal ILM could be successfully extracted and conserved on only three occasions (samples 1, 3, and 6). In three further samples, the central segment was preserved but presented with a central aperture, so that the immediate foveal region could not be examined. In most segments, either the retinal or the vitreal side was consistently exposed, whereas in some quadrants ILM fragments were folded, with areas of both retinal and vitreal side facing upward. ILMs with the vitreal side up (retinal side attached to the glass substrate) showed a higher tendency to separate from the substrate, which explains why more ILM segments with the retinal side exposed were available for AFM measurements.

A total of 39 segments from 10 donor eyes underwent AFM thickness measurements, including measurements from both the vitreal and the retinal side. AFM stiffness measurements were performed by means of 37 segment analyses from nine donor eyes, including 29 from the retinal and 8 from the vitreal side (Table 1).

\section{TEM Measurements}

For TEM, standard procedures were followed as described earlier. ${ }^{3}$ Briefly, samples were fixed in $2.5 \%$ glutaraldehyde and $2.5 \%$ paraformaldehyde overnight. The samples were osmicated and
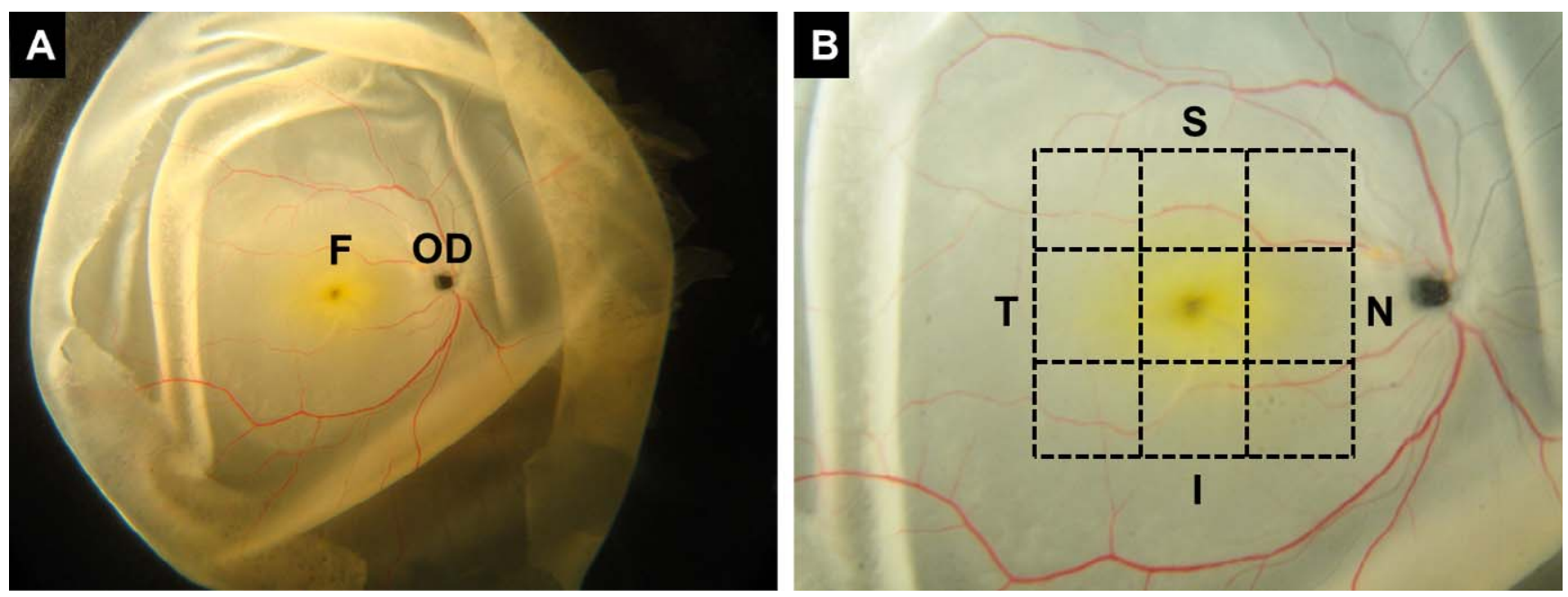

FiguRE 1. (A) Human donor retina. OD, optic disc; F, fovea. (B) Zoom-in of (A) with plot of posterior pole grid. N, nasal; S, superior; T, temporal; I, inferior. 
TABle 1. Overview of Basic Donor Characteristics

\begin{tabular}{|c|c|c|c|c|c|c|c|c|}
\hline \multirow[b]{2}{*}{ Sample No. } & \multirow[b]{2}{*}{ Donor age } & \multirow[b]{2}{*}{ Gender } & \multirow[b]{2}{*}{$\begin{array}{l}\text { Cause of } \\
\text { death }\end{array}$} & \multirow[b]{2}{*}{$\mathrm{OD} / \mathrm{OS}$} & \multirow[b]{2}{*}{$\begin{array}{l}\text { Underlying } \\
\text { pathology }\end{array}$} & \multicolumn{3}{|c|}{ \# Measured segments } \\
\hline & & & & & & Thickness & $\begin{array}{l}\text { Stiffness } \\
\text { retinal side }\end{array}$ & $\begin{array}{c}\text { Stiffness } \\
\text { vitreal side }\end{array}$ \\
\hline 1 & 52 & $\mathrm{~m}$ & Peritonitis & OS & $\begin{array}{c}\text { IDDM } \times 17 y, \\
\text { HTN, } s / \mathrm{p} \\
\text { CABG, } \\
\text { medication- } \\
\text { induced renal } \\
\text { failure, } s / \mathrm{p} \\
\text { carotid } \\
\text { edarterectom } \\
y\end{array}$ & 1 & 1 & 0 \\
\hline 2 & 54 & $\mathrm{~m}$ & Cardiac arrest & OS & $\begin{array}{l}\text { HIM since } \\
\text { childhood }\end{array}$ & 5 & 2 & 2 \\
\hline 3 & 62 & $\mathrm{~m}$ & $\mathrm{PE}$ & OS & $\begin{array}{c}\text { CABG, IDDM x } \\
15 \mathrm{y} \\
\text { Pseudophakia }\end{array}$ & 1 & 1 & 0 \\
\hline 4 & 66 & $\mathrm{~m}$ & $\mathrm{ICH}$ & OD & $\begin{array}{c}\text { CAD, HTIN, } \\
\text { seizures, } \\
\text { dementia }\end{array}$ & 7 & 7 & 0 \\
\hline 5 & 66 & $\mathrm{~m}$ & $\mathrm{ICH}$ & OS & $\begin{array}{c}\text { CAD, HTIN, } \\
\text { seizures, } \\
\text { dementia }\end{array}$ & 9 & 9 & 0 \\
\hline 6 & 72 & $f$ & $\mathrm{ICH}$ & OS & $\begin{array}{c}\text { HTN } \times 5 \text { years, } \\
\text {, previous MI, } \\
\text { COPD, RA, } \\
\text { renal stones }\end{array}$ & 1 & 0 & 0 \\
\hline 7 & 80 & $\mathrm{~m}$ & Acute MI & OD & $\begin{array}{c}\text { HTN } \times 5 \text { years, } \\
\text { Pacemaker } \times 5 \\
y, \text { GERD }\end{array}$ & 3 & 0 & 3 \\
\hline 8 & 81 & $f$ & $\begin{array}{c}\text { Traffic } \\
\text { accident }\end{array}$ & $O D$ & $\begin{array}{c}\text { Emphysema, } \\
\mathrm{Cl} \text {, Peripheral } \\
\text { Intraretinal } \\
\text { hemorrhages }\end{array}$ & 7 & 6 & 3 \\
\hline 9 & 82 & $\mathrm{~m}$ & Cardiac arrest & OS & $\begin{array}{c}\text { PTIN } \times 45 \\
\text { years, CVA, } \\
\text { S/p CABG, } \\
\text { COPD } \times 5 y \text { y, } \\
\text { DVT }\end{array}$ & 4 & 2 & 0 \\
\hline 10 & 83 & $\mathrm{~m}$ & CVA & OS & $\begin{array}{c}\text { CABG, HTN, } \\
\text { Internal } \\
\text { defibrillator, } \\
\text { PVD, BPH, OA }\end{array}$ & 1 & 1 & 0 \\
\hline
\end{tabular}

BPH, benign prostatic hypertrophy; CAD, coronary artery disease; CABG, coronary artery bypass graft; CI, cochlear implants; COPD, chronic obstructive pulmonary disease; CVA, cerebrovascular accident; DVT, deep vein thrombosis; GERD, gastroesophageal reflux disease; HM, heart murmur; HTN, arterial hypertension; ICH, intracerebral hemorrhage; IDDM, insulin dependent diabetes mellitus; MI, myocardial infarction; OA, osteoarthritis; PE, pulmonary embolism; PVD, peripheral vascular disease; RA, rheumatoid arthritis. 
embedded in EPON (Embed 812, Electron Microscopy Sciences, Hatfield, PA). Thin sections were examined under a JEOL electron microscope (JEOL GmBH, Eching, Germany) at $\times 25,000$.

TEM images from 13 retinas from donors aged 54 to 88 years (mean 75 years) were analyzed using Adobe Photoshop CS5 Edition (Adobe Systems, Inc., Los Altos, CA). For each retina, the mean pixel density per $250 \times 250-\mathrm{nm}$ area was determined in at least two images for the most vitreal and the most retinal ILM layers using the inversion function of the program. The mean was calculated and plotted.

\section{AFM Measurements}

All AFM imaging and force indentation experiments were performed using a Nanowizard I Atomic Force Microscope (JPK Instruments, Berlin, Germany) and a FlexAFM "ARTIDIS" (Nanosurf AG, Liestal, Switzerland), which were mounted on a Zeiss Axiovert $135 \mathrm{TV}$ and Zeiss Axiobserver A1 inverted microscopes (Zeiss AG, Oberkochen, Germany) respectively.

For imaging and stiffness measurements with Nanowizard I AFM, standard silicon-nitride triangular cantilevers (DNP-S10, Veeco, Santa Barbara, CA) with integrated sharp silicon-nitride pyramidal tips (nominal spring constant $\mathrm{k}=0.06 \mathrm{~N} / \mathrm{m}$, radius $\mathrm{r} \sim 10 \mathrm{~nm}$ ) were used. For imaging with FlexAFM "ARTIDIS," standard silicon-nitride rectangular cantilevers (HYDRA6R-50NG, AppNano, Santa Clara, CA) with integrated silicon tips (nominal spring constant $\mathrm{k}=0.08 \mathrm{~N} / \mathrm{m}$, radius $\mathrm{r}$ $\sim 8 \mathrm{~nm}$ ) were used. Before every measurement, the spring constant value was determined with the thermal fluctuation method. ${ }^{24}$ The samples were imaged in contact mode with a scan rate of $0.8 \mathrm{~Hz}$ while kept in PBS at room temperature.

ILM thickness was determined from AFM height images. A height profile was calculated based on the glass surface reference as described previously. ${ }^{3}$ Two scan regions of $100 \times 100 \mu \mathrm{m}$ were randomly chosen within each grid segment. Height data of these scan regions were gathered in a histogram and averaged.

To plot a thickness profile of a larger, contiguous ILM area around the fovea centralis, a series of AFM images was measured, beginning in the actual center of the fovea centralis. A discrete overlap of each scan with the preceding one was ensured. Small lacerations with a diameter of $165 \mu \mathrm{m}$ and $600 \mu \mathrm{m}$ (samples 8 and 10, respectively) in the immediate center of the fovea allowed the use of the exposed glass substrate as reference planes (Table 1). The "multidata merge" function of the Gwyddion software package (version 2.17, Czech Metrology Institute, Brno, Czech Republic) was used to align neighboring images to the reference plane of the central image and generate image series (Fig. 2B). Measurements for the image series were performed from the retinal side.

Stiffness was determined by using AFM in a force spectroscopy mode as described previously. ${ }^{25}$ Briefly, indentations were made over a $20 \times 20$-point grid at a rate of one load/unload cycle per second with a maximal load of $3.1 \mathrm{nN}$. Each force curve consisted of 512 data points. Assessment of the dynamic indentation modulus $|\mathrm{E}|$ of each forceindentation curve was performed off-line by using custom-made software (LabVIEW; National Instruments, Austin, TX) based on Oliver and Pharr's method. ${ }^{26}$ Irreversible deformation or other extraneous displacement effects were avoided by using unloading (retraction) rather than loading curves (Fig. 3).

\section{Immunostaining}

ILM samples were stained for both laminin (L9393; Sigma) and collagen $\mathrm{IV}^{27}$ for better visualization of the membrane. The laminin antibodies that were used represent polyclonal rabbit laminin antibodies directed against murine Engelbreth-Holm-Swarm (EHS) sarcoma laminin. The staining of human ILM with this antiserum was very similar to that obtained with a monoclonal antibody to laminin $\alpha 5$, the $\alpha$ chain most abundant in adult human ILM. ${ }^{28}$ The polyclonal rabbit antibody to collagen IV was raised against collagen IV from human amnion. ILM staining with this antibody was very similar to the staining obtained using rat monoclonal antibodies to collagen IV $\alpha 3$ and $\alpha 5,{ }^{29}$ the most abundant collagen IV chains in human ILM. ${ }^{30}$ The primary antibodies were applied for 1 hour, after which the slides were washed three times with PBS. Secondary antibodies (Alexa 488 and $\mathrm{Cy} \alpha, \mathrm{c}=1: 800$ in PBS) were then added for 1 hour each, after which the slides were rinsed again three times.

\section{Results}

\section{Morphology of Native Human ILM}

TEM images revealed an asymmetrical appearance of ILM surfaces with a smooth vitreal side and an irregular retinal side, marked by invaginations and recesses in immediate apposition to underlying retinal structures (Figs. 4A, 4B). AFM results confirmed a characteristic ILM orientation with dissimilar surfaces. The vitreal side showed a smooth surface aspect (Fig. $4 \mathrm{C}$ ), whereas the retinal side was marked by a conspicuous bold relief with structures appearing like mountains and ridges separated by valleys and crypts (Fig. 4D).

Mean thickness measurements acquired from opposite ILM orientations showed similar results (Fig. 4E, $3707 \pm 0.061 \mathrm{~nm}$ on the retinal versus $3826 \pm 0.256 \mathrm{~nm}$ on the vitreal side; $P=$ 0.232 ), but standard deviation and roughness (RMS) values were higher on the retinal side (Fig. 4F).

Retinal side thickness values were relatively evenly distributed throughout the midperiphery, with segment averages ranging from $3229 \mathrm{~nm}$ to $3663 \mathrm{~nm}$, and a mean value of 3340 $\pm 436 \mathrm{~nm}$. Thickness within the central segment was higher, averaging $3987 \pm 216 \mathrm{~nm}(P=0.001$, Mann-Whitney $U$ test $)$. Average retinal side thickness over all segments yielded 3488 $\pm 460 \mathrm{~nm}$.

\section{Characterization of the Fovea Centralis}

Thickness measurements within the central $1000 \mu \mathrm{m}$ were possible in three eyes, where the delicate foveal ILM was preserved throughout the extraction and flat-mounting process. AFM analysis revealed four distinct layers separated by comparatively steep thickness increments. The first layer was detected within the central $400 \mu \mathrm{m}$ from the foveal center, where average ILM thickness was $138 \pm 80 \mathrm{~nm}$ with an RMS roughness of $34.2 \mathrm{~nm}$ (Fig. 2). The second layer was observed beginning at $400 \mu \mathrm{m}$ up to $600 \mu \mathrm{m}$ from the center of the fovea and displayed a mean thickness of $567 \pm 494 \mathrm{~nm}$ and an RMS roughness of $155.14 \mathrm{~nm}$. The third layer began at a distance of 600 to $900 \mu \mathrm{m}$ from the center and showed a mean thickness of $1921 \pm 516 \mathrm{~nm}$ and an RMS roughness of $256.4 \mathrm{~nm}$. Peak ILM thickness values averaging $3711 \pm 300 \mathrm{~nm}$ and displaying an even higher RMS roughness of $510.6 \mathrm{~nm}$ were found at a distance of 900 to $1050 \mu \mathrm{m}$ from the center of the fovea.

The difference between the average values from measurements on dehydrated ILMs and native ILMs is particularly salient in the center of the fovea (native ILM 6.9 times thicker than dehydrated specimens) and still considerable at the extrafoveal posterior pole (native ILM 1.5 times thicker). Immediate comparison of more peripheral ILM thickness results was not possible, as for midperipheral segments no corresponding values were found in the literature, whereas measurements for the anterior retina were not performed in the current study.

\section{Nanomechanical Properties of the ILM}

Indentation analysis of the retinal side yielded an overall mean stiffness of $224.6 \pm 43.2 \mathrm{kPa}$. Stiffness was significantly higher in the central quadrant $(276 \pm 46 \mathrm{kPa})$ compared with the 

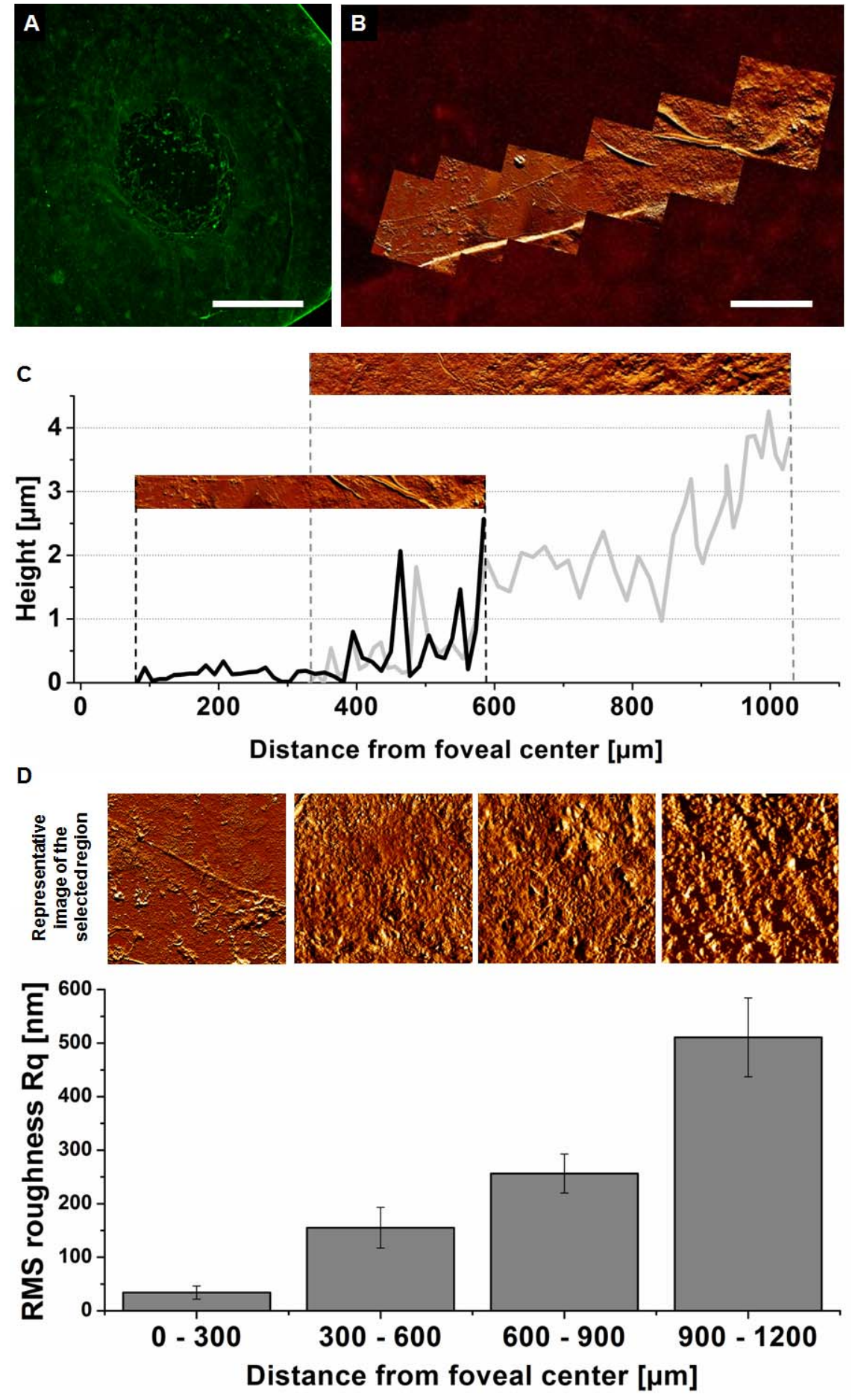

Figure 2. (A) Immunofluorescence image of the ILM from sample 4 central quadrant, retinal side aspect. Green channel (laminin). Scale bar $=500$ $\mu \mathrm{m}$. The ILM region overstretching the fovea centralis is clearly visible and shows a peculiar craterlike architecture with centrifugally increasing thickness and surface roughness. (B) Immunofluorescence image from sample 8. Red channel (collagen IV). Scale bar $=100 \mu \mathrm{m}$. ILM overlying the fovea centralis features a laceration in the immediate center, which served as a reference plane for alignment of multiple contiguous AFM scans, allowing the calculation of a thickness profile across the foveal region. (C) The ILM shows an acute increase in both thickness and surface irregularity in the foveal region. Measurements and images depict the retinal surface. Black line: Sample 8. Gray line: Sample 10. (D) Increase of average RMS roughness values (gray columns) $+\mathrm{SD}$ (error bars) correlates with distance from the fovea centralis. Each image represents $80 \times 80 \mu \mathrm{m}$.

surrounding midperipheral segments $(217.2 \pm 33.3 \mathrm{kPa} ; P=$ 0.04) (Fig. 5). Measurements of ILM stiffness in the immediate foveal center were not possible, as the thinness of the foveal ILM did not allow for reliable measurements owing to potential substrate artifacts.
Indentation measurements of the vitreal side of the central segment were not included in the analysis because of the small amount of available samples. Vitreal side stiffness measurements of the five available midperipheral segments averaged 44 $\pm 4 \mathrm{kPa}$. Compared with the retinal side, the vitreal side proved 

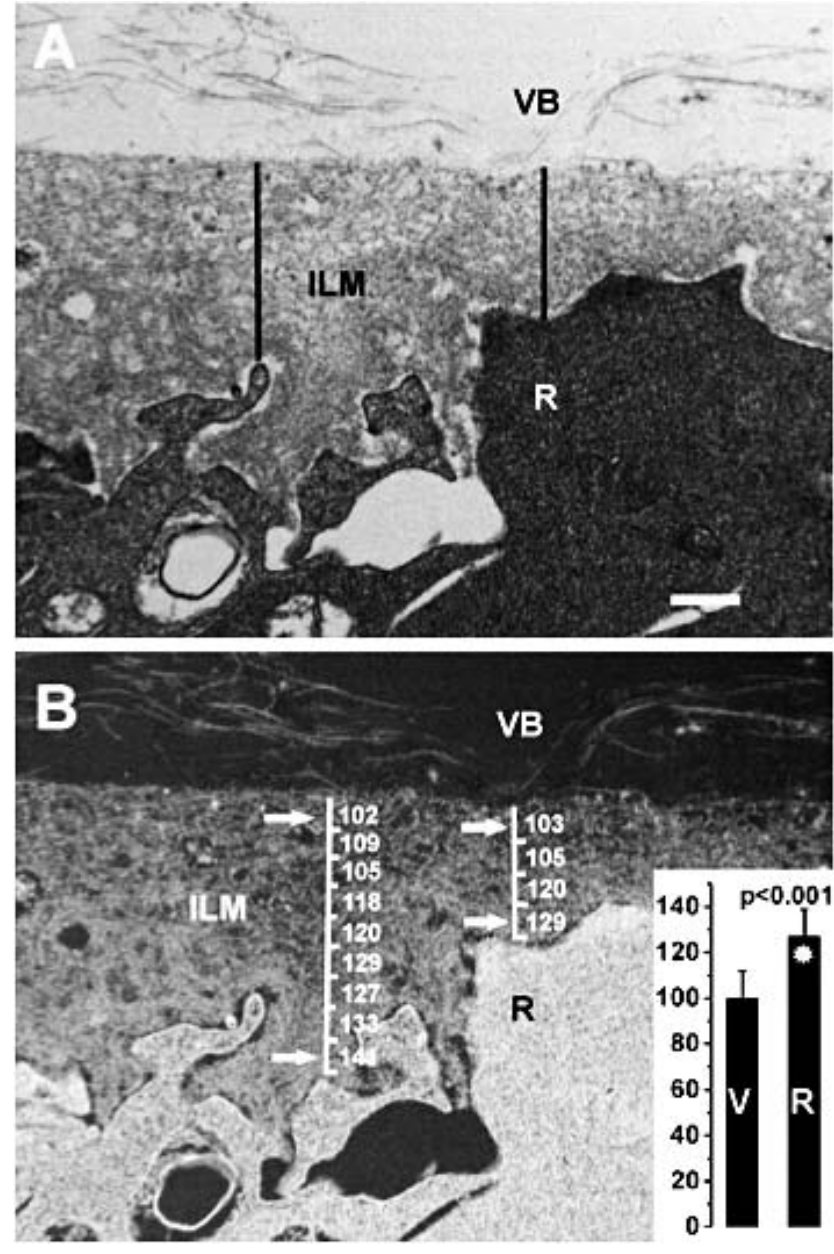

Figure 3. TEM image of an 80-year-old ILM in situ (A) illustrating a higher density of ECM protein at the vitreal versus the retinal surface. The image $(\mathbf{B})$ was inverted and the mean pixel density per $250 \times 250$ $\mathrm{nm}$ unit areas was determined throughout the thickness of two segments of the ILM. The graph in (B) compares the mean pixel density for the retinal (R) and the vitreal (V) part (as indicated by arrows in panel B) of the ILM from 13 retinas. R, retina; VB, vitreous body. Scale bar $=500 \mathrm{~nm}$.

4.9 times softer over all available midperipheral segments. In three midperipheral segments, stiffness measurements of both the retinal and the vitreal sides were possible in identical tissue owing to folding of the ILM. Average ratio between retinal and vitreal side stiffness in these segments was 3.83 (sample 8: superior central segment: 3.74; medial temporal segment: 4.17; sample 2: inferior central segment: 3.57 ).

It was noted that the density of ECM proteins of the vitreal part of the ILM was consistently lower than the density of the retinal part (Fig. 3A). For quantification, the TEM images from 13 retinas from donors ranging from 54 to 88 years of age were inverted and the mean pixel density at the retinal and vitreal layers of the ILM was determined. It was found that the density was $20 \%$ higher for the retinal layer as compared with the vitreal layer. The difference was statistically significant $(P<$ 0.001, Fig. 3B).

\section{Discussion}

Although AFM has been available for more than 2 decades, ${ }^{31}$ it has been underused in vision sciences. ${ }^{32}$ To the best of our knowledge, a comprehensive description of topographic and nanomechanical variations of the native human ILM has not been undertaken. A previous study described reduced ILM stiffness in Protein Omannose N-acetylglucosaminyltransferase 1 knockout mice (POMGnT1), one of the mouse models of muscular dystrophy, implicating a deficiency of POMGnT1mediated glycosylation of dystroglycan. ${ }^{33}$ Candiello et al. ${ }^{3}$ described an age-dependent increase in ILM thickness and stiffness in specimens taken from outside the posterior pole of a series of human donor eye retinas. A recent AFM examination of freshly harvested surgically removed human ILMs from diabetic patients with macular holes focused on surface adhesion forces determined by different ILM surface patterns. "Globular structures" were described on the surface of all specimens, whereas "fibrillar structures" on only a small number of samples were associated with higher adhesion forces. Although the study generally supports the suitability of AFM for studying the ILM, it is important to note that measurements were performed on fixed samples stored at $-20^{\circ} \mathrm{C}$, whereas the fundamental advantage of AFM over the more commonly used TEM is that native samples can be examined under physiologic buffer conditions. ${ }^{32}$ Available topographic information from light and TEM examinations may, in fact, not accurately portray the native ILM, as the corresponding fixation process involves tissue dehydration and a consequent loss of water-binding GAG side chains of ILM proteoglycans. Comparative TEM and AFM measurements of ILM samples harvested outside the great temporal vascular arcades have shown that a dehydration step in the fixation procedure induces $30 \%$ to $50 \%$ reduction in ILM thickness and $30 \%$ increase in stiffness. ${ }^{3}$ In contrast with these earlier AFM measurements, the present study is particularly clinically relevant, as it provides a detailed description of ILM topography and biomechanics of the clinically highly meaningful posterior pole. This area within the great vascular arcades includes the location of the fovea centralis, a small morphologically distinct part of the retina with markedly enhanced visual resolution. The posterior pole is also characteristically the location where vitreomacular adhesions lead to a variety of pathologies and where surgical removal of the ILM is performed during chromovitrectomy.

Initial TEM analyses of the vitreoretinal interface showed close apposition of the ILM to the underlying inner retinal layers, as well as a characteristic ILM orientation with dissimilar surfaces (Figs. 4A, 4B). These findings were confirmed by AFM measurements (Figs. 4C, 4D). Although AFM thickness measurements from both sides of the ILM expectably resulted in similar average values, measurements from the retinal surface displayed a higher standard deviation and RMS than measurements from the vitreal side (Fig. 4E), consistent with the roughness of the retinal surface.

ILM thickness appeared rather evenly distributed throughout the midperipheral segments (Fig. 6), whereas a closer look at the central sector revealed a conspicuous ILM gauge distribution. The ILM overlying the fovea centralis could be imaged by AFM at unprecedented detail. Its craterlike aspect with partially round-arched, relatively steep walls translated into a centrifugally increasing thickness profile (Fig. 2A), reaching a maximum at a foveal distance of approximately $1000 \mu \mathrm{m}$, from where a gradual drop-off was observed (Fig. 2C). This gradual decline extended toward the more peripheral grid segments.

In fact, there seems to be a quadrinomial division of the foveal ILM structure, where additional layers appear to be ramped up on a smooth, 138-nm-thick substratum, which marks the central 350 to $400 \mu \mathrm{m}$. This central-most part of the ILM displays a smooth aspect even when imaged from the retinal side. Immediately outside this most central zone, a steep 

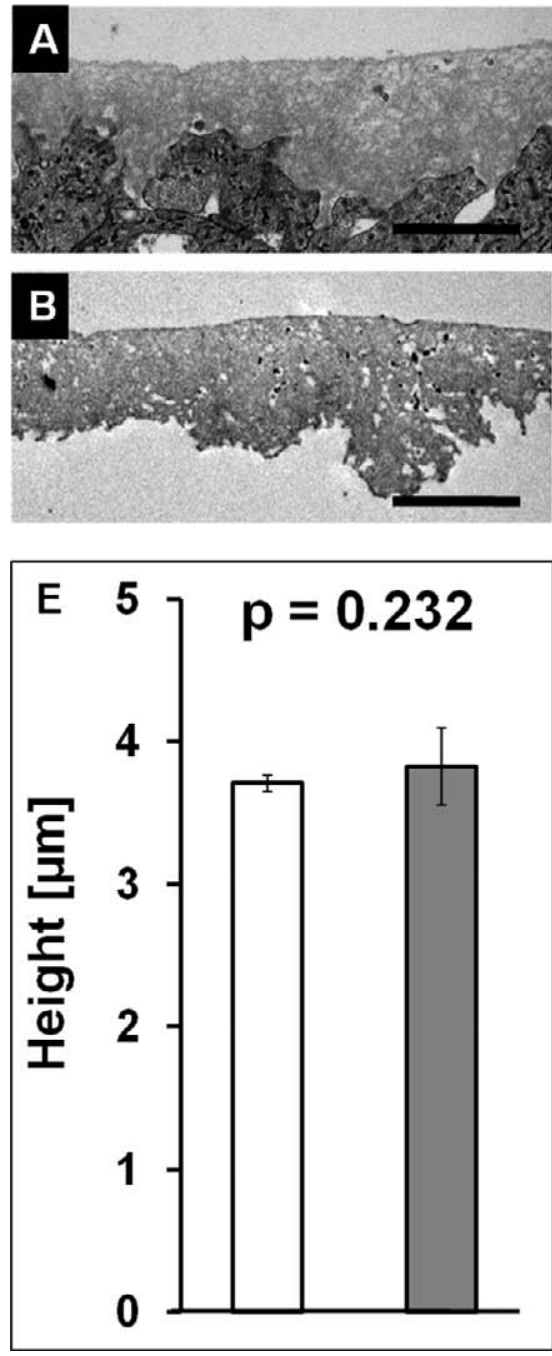
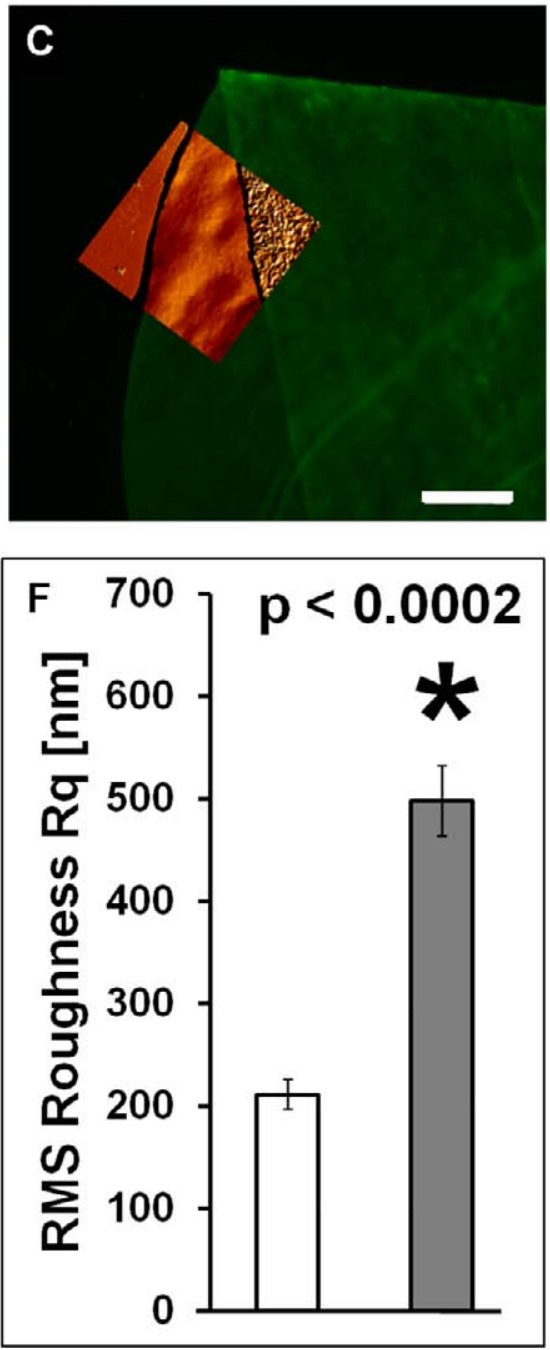
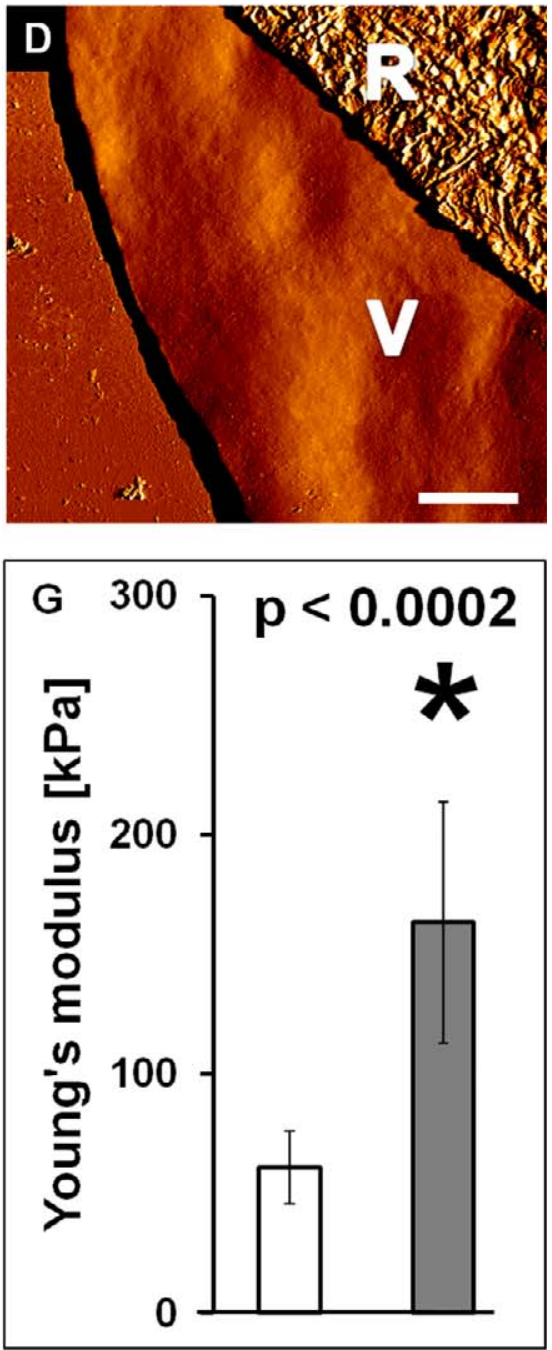

Figure 4. (A) TEM image of ILM with adjacent retina. Scale bar $=1 \mu \mathrm{m}$. (B) TEM image of ILM without retina. Scale bar $=1 \mu \mathrm{m}$. (C) Fluorescence image of a representative ILM stained for laminin with an overlapping AFM scan in the center. Scale bar $=50 \mu \mathrm{m}$. (D) Enlarged AFM scan from (C). Right top: Retinal surface (R). Center: Vitreal surface (V). Left: Substrate. Scale bar $=10 \mu \mathrm{m}$. (E) Average ILM thickness of a single representative sample (white column: vitreous-facing side; grey column: retina-facing side) + SD (error bars). (F) Average RMS values of vitreal (white) and retinal (gray) facing sides + SD (error bars). Sampled surface area $=20 \times 20 \mu \mathrm{m}^{2}, \mathrm{n}=5$. (G) Average stiffness values of force-indentation measurements from both vitreal (white) and retinal (gray) sides of the ILM + SD (error bars).

thickness increase to about $750 \mathrm{~nm}$ can be observed at a foveal distance of roughly $600 \mu \mathrm{m}$, followed by a second and a third relatively abrupt increase to about $1500 \mu \mathrm{m}$ at a foveal clearance of 850 to $900 \mu \mathrm{m}$ and to $4000 \mathrm{~nm}$ at 1000 to 1050 $\mu \mathrm{m}$, respectively. Whether these offsets represent distinct additional tissue layers is yet to be explored in future research.

External to the immediate foveal region, the increase in ILM thickness is accompanied by progressive increase in retinal surface roughness, suggesting distinct extracellular matrix layers spread on a basic stratum, which, based on surface appearance and roughness analysis, is contiguous with the vitreal surface of the ILM (Figs. 2A, 2D). To the best of our knowledge, this continuous vitreal side basic stratum has not been described before. Thickness distributions closely match earlier ILM thickness findings, ${ }^{8}$ although ILM thickness under in vivo conditions is roughly four times higher than previously suggested based on LM and TEM analyses ${ }^{7,8}$ and consistent with previous AFM examinations of human ILM segments from outside the posterior pole. ${ }^{3}$

Analyses of ILM stiffness matched thickness findings with respect to geographical distribution, with higher stiffness values in the central segment compared with the midperipheral quadrants. As ILM stiffness facilitates lifting up an initial ILM flap during surgical ILM removal, these findings underscore that ILM peeling may be most easily commenced within a foveal distance of roughly $1000 \mu \mathrm{m}$. Unlike for ILM thickness, a detailed distribution of ILM stiffness values within the central quadrant could not be specified, as reliable measurements of the extremely delicate foveal center were not possible in this study, owing to unavoidable underlying glass substrate influence.

ILM stiffness, on average, is 4.93 times higher on the retinal than on the vitreal side. Although sample size was narrowed by a higher rate of tissue loss throughout the preparation process for ILM mounted with the vitreal side up, we consider the results valid, especially as they could be corroborated through measurements on three tangled samples where measurements from the retinal and vitreal sides were possible on identical tissue (retinal side 3.83 times stiffer).

In line with this bipolar stiffness distribution, a significantly higher ECM density was found in the retinal compared with the vitreous layers of the ILM (Fig. 3). Apart from ECM density, 


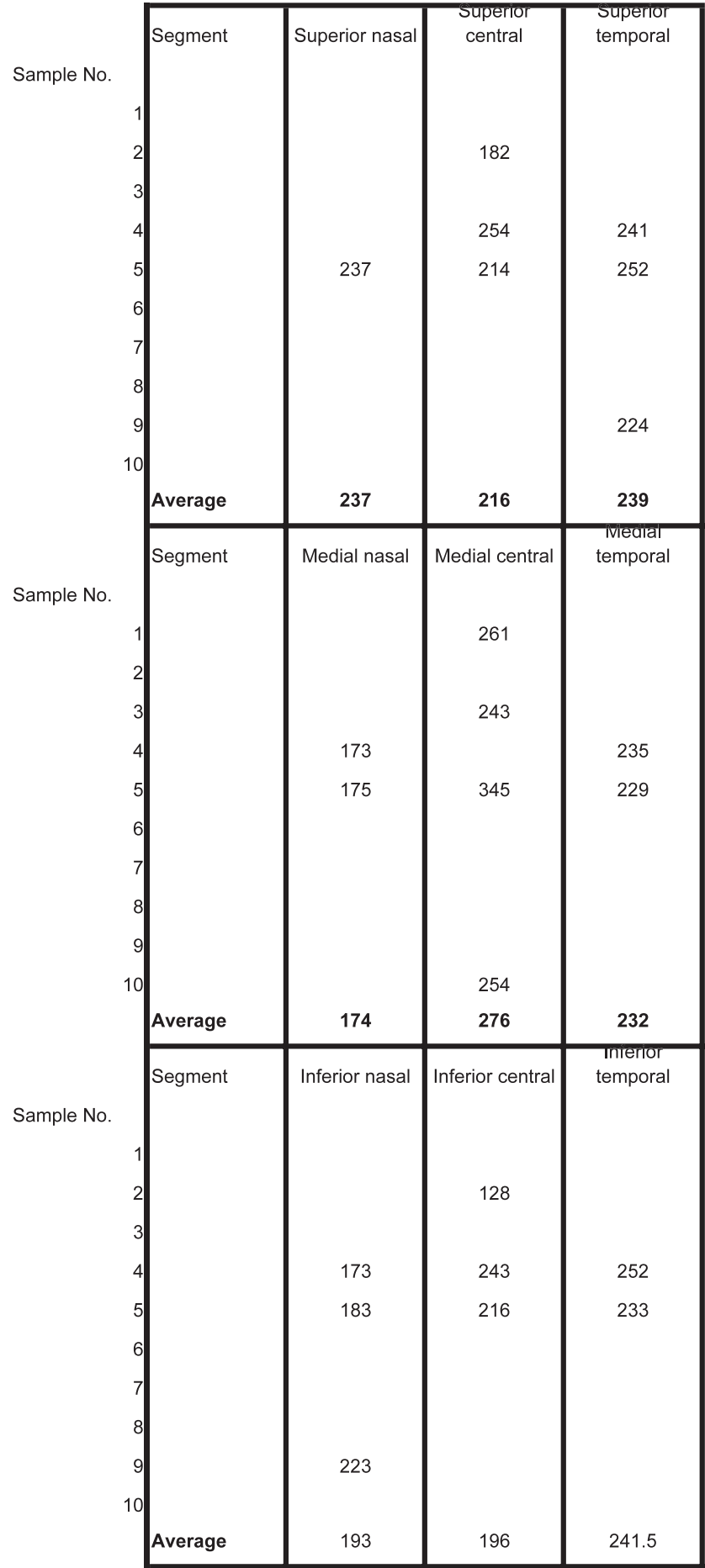

FIgURE 5. Retinal side stiffness values in $\mathrm{kPa}$ are displayed for each sample by segment. Samples are indicated in the ordinate. Each individual value represents an average of all height values collected from two distinct scan areas of $100 \times 100 \mu \mathrm{m}$ each. Blank cells denote samples lost during the preparation process. At the bottom of each segment, the overall average for all measurements within the segment is indicated.

ILM stiffness disparity may also be influenced by protein variety, protein isoform composition, ${ }^{34,35}$ osmotic pressure, ${ }^{36}$ and the density of cross-links. ${ }^{37}$ As the ILM is composed of a distinct and limited set of chains for both collagen IV and laminin, immunostaining of the ILM may have been influenced

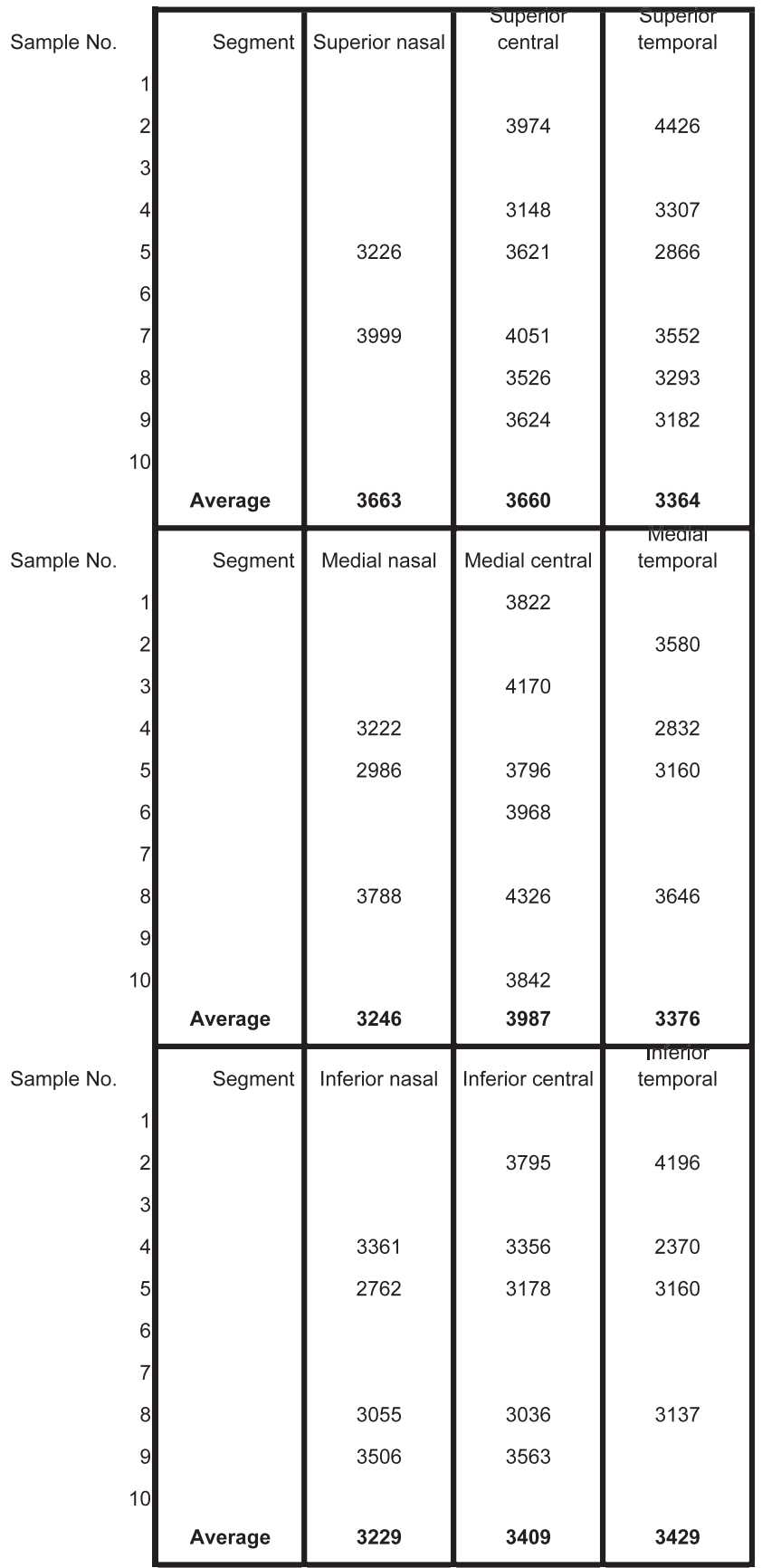

Figure 6. ILM thickness values in $\mathrm{nm}$ are displayed for each donor sample by segment. Samples are indicated in the ordinate and ordered by age $(1=$ youngest patient, $10=$ oldest patient, compare with Table 1). Each individual value represents an average of all height values collected from two distinct scan areas of $100 \times 100 \mu \mathrm{m}$ each. Blank cells denote samples lost during the preparation process. At the bottom of each segment, the overall average for all measurements within the segment is indicated.

by the specificity of the antibodies used for certain isoforms of both proteins.

The vitreoretinal interface represents the site of apposition of the posterior cortical vitreous and the ILM, and, thus, plays a key role in physiologic and pathologic vitreomacular adhesion. Over the past decade, chromovitrectomy has become a standard treatment for diseases based on pathologic vitreomacular adhesion, including macular holes, persistent macular 
edema, epiretinal fibrosis, and vitreomacular traction syndrome. ${ }^{11,38-41}$ This surgical technique, which involves surgical removal of the ILM following intravitreal application of vital dyes, intended to improve ILM visibility, has evolved from accidental excision of parts of the ILM during epiretinal membrane removal. ${ }^{42}$ ICG is the most commonly used vital dye, although this substance is not approved for intravitreal use and despite significant evidence for retinal and optic nerve toxicity. ${ }^{43}$ ICG has been described to facilitate the excision of the ILM by means of an ILM stiffening effect through collagen IV cross-linking, ${ }^{20}$ which might contribute to its popularity among vitreoretinal surgeons. This effect on the mechanical properties of the ILM, however, has not been firmly established. The high-resolution map of healthy ILM nanoscale topography and stiffness provided in the current study contributes groundwork anatomic and physiologic information that will allow future study of the effect of vital dyes on biomechanical properties of the ILM. Preliminary results from freshly harvested patient ILMs had resulted in a high degree of variability. Interpretation of the data was not possible without detailed knowledge of mechanical characteristics of the native ILM. As the current study shows, consideration of the exact location from which a certain fragment of ILM was extracted and of the orientation from which it was measured (vitreal versus retinal side) are indispensable for data interpretation (Henrich et al., manuscript in preparation).

The thickness profile reported in this study also suggests that best staining results during chromovitrectomy can be expected at a foveal offset of roughly $1000 \mu \mathrm{m}$, owing to increased thickness, as well as favorable conditions for grasping of the ILM with intraocular forceps, although these theoretical advantages need to be weighed with foveal proximity in practice.

The results of this study are also in accordance with the current concept of macular hole formation, ${ }^{44}$ involving the peculiar fragility and thinness of the foveal retina in combination with the insertion of native vitreal collagen fibrils into the collagen network of the foveal ILM. The ILM has been described to be the main contributor to mechanical stability of the retina and vitreoretinal border. ${ }^{45}$ The particular frangibility of the foveal ILM can, thus, be regarded as a fundamental factor for the formation of macular holes. Fragility of the foveal ILM is underscored by the fact that conservation of the central-most ILM was possible in only in three specimens in this study.

Finally, the vitreous cavity is a favorite site for delivering therapeutic antibodies, cDNAs, and viruses into the eye and retina. The ILM represents the major border that prevents ready access of the reagents to the retinal tissue. It is conceivable that differences in ILM thickness and stiffness, as measured here, have a major effect on the success of drug delivery to retinal cells. ${ }^{46}$

\section{Conclusions}

A detailed topographical and nanomechanical profile of native human ILMs was generated using AFM. The thickness of the native ILM averages to $3488 \pm 460 \mathrm{~nm}$. It is, thus, roughly four times thicker than previously described in TEM analyses. Detailed structural analysis confirmed a topographic variation of ILM thickness, notably around the fovea centralis: The foveal ILM displayed a craterlike morphological appearance with four distinct layers separated by comparatively steep thickness increments. Additional layers appear to be ramped up on a smooth basic substratum marking the central 350 to $400 \mu \mathrm{m}$. Based on morphology and roughness, this basic stratum is contiguous with the vitreal surface of the ILM. Thickness is minimal in this immediate center of the fovea $(138 \pm 80 \mathrm{~nm})$ and peaks to more than $4000 \mathrm{~nm}$ at a foveal distance of roughly $1000 \mu \mathrm{m}$. Retinal side stiffness values also show a spatial distribution, with higher values in the central segment (276 \pm $46 \mathrm{kPa})$ compared with the midperiphery $(217.2 \pm 33.0 \mathrm{kPa})$. ILM stiffness is approximately five times higher on the retinal side (average $224.6 \pm 43.0 \mathrm{kPa}$ ) than on the vitreal side ( $44 \pm$ $4 \mathrm{kPa}$ ). Interrelated TEM and AFM analysis allowed a correlation of the heterogeneous stiffness distribution with higher ECM density on the retinal than on the vitreal side. AFM opens new possibilities for investigating structure function relationships of native basement membranes in physiological and pathological environment.

\section{References}

1. Balasubramani M, Schreiber EM, Candiello J, Balasubramani GK, Kurtz J, Halfter W. Molecular interactions in the retinal basement membrane system: a proteomic approach. Matrix Biol. 29:471-483.

2. Erickson AC, Couchman JR. Still more complexity in mammalian basement membranes. J Histochem Cytochem. 2000;48:1291-1306.

3. Candiello J, Cole GJ, Halfter W. Age-dependent changes in the structure, composition and biophysical properties of a human basement membrane. Matrix Biol. 29:402-410.

4. Halfter W, Winzen U, Bishop PN, Eller A. Regulation of eye size by the retinal basement membrane and vitreous body. Invest Ophthalmol Vis Sci. 2006;47:3586-3594.

5. Timpl R, Brown JC. Supramolecular assembly of basement membranes. Bioessays. 1996;18:123-132.

6. Hogan MJ. The vitreous, its structure, and relation to the ciliary body and retina. Proctor Award Lecture. Invest Opbthalmol. 1963;2:418-445.

7. Fine BS. Limiting membranes of the sensory retina and pigment epithelium. An electron microscopic study. Arch Ophthalmol. 1961;66:847-860.

8. Foos RY. Vitreoretinal juncture; topographical variations. Invest Ophthalmol. 1972;11:801-808.

9. Matsumoto B, Blanks JC, Ryan SJ. Topographic variations in the rabbit and primate internal limiting membrane. Invest Opbthalmol Vis Sci. 1984;25:71-82.

10. Valentin-Rodriguez C, Tezel TH, Ivanisevic A. Quantitative analysis of human internal limiting membrane extracted from patients with macular holes. Langmuir. 26:12810-12816.

11. Gandorfer A, Messmer EM, Ulbig MW, Kampik A. Resolution of diabetic macular edema after surgical removal of the posterior hyaloid and the inner limiting membrane. Retina. 2000;20: 126-133.

12. Gandorfer A, Ulbig M, Kampik A. Plasmin-assisted vitrectomy eliminates cortical vitreous remnants. Eye (Lond). 2002;16: 95-97.

13. Al-Abdulla NA, Thompson JT, Sjaarda RN. Results of macular hole surgery with and without epiretinal dissection or internal limiting membrane removal. Opbthalmology. 2004;111:142149.

14. Smiddy WE, Feuer W, Cordahi G. Internal limiting membrane peeling in macular hole surgery. Opbthalmology. 2001;108: 1471-1476; discussion 1477-1478.

15. Haritoglou C, Gass CA, Schaumberger M, Gandorfer A, Ulbig MW, Kampik A. Long-term follow-up after macular hole surgery with internal limiting membrane peeling. Am J Opbthalmol. 2002;134:661-666.

16. Haritoglou C, Schuttauf F, Gandorfer A, Thaler S. An experimental approach towards novel dyes for intraocular surgery. Dev Opbthalmol. 2008;42:141-152. 
17. Rodrigues EB, Penha FM, Farah ME, et al. Preclinical investigation of the retinal biocompatibility of six novel vital dyes for chromovitrectomy. Retina. 2009;29:497-510.

18. Morales MC, Freire V, Asumendi A, et al. Comparative effects of six intraocular vital dyes on retinal pigment epithelial cells. Invest Ophthalmol Vis Sci. 2010;11:6018-6029.

19. Wollensak G, Sporl E, Pham DT. Biomechanical changes in the anterior lens capsule after trypan blue staining. J Cataract Refract Surg. 2004;30:1526-1530.

20. Wollensak G, Spoerl E, Wirbelauer C, Pham DT. Influence of indocyanine green staining on the biomechanical strength of porcine internal limiting membrane. Ophthalmologica. 2004; 218:278-282.

21. Lundkvist A, Reichenbach A, Betsholtz C, Carmeliet P, Wolburg $\mathrm{H}$, Pekny M. Under stress, the absence of intermediate filaments from Müller cells in the retina has structural and functional consequences. J Cell Sci. 2004;117:3481-3488.

22. Plodinec M, Loparic M, Aebi U. Atomic force microscopy for biological imaging and mechanical testing across length scales. Cold Spring Harb Protoc. 2010;2010:pdb top86.

23. Plodinec MLM, Aebi U. Force Microscopy for Biological Imaging and Mechanical Testing across Length Scales, in Live Cell Imaging: A Laboratory Manual. Cold Spring Harbor: Cold Spring Harbor Laboratory Press; 2010.

24. Hutter JLBJ. Calibration of atomic-force microscope tips. Rev Sci Instrum. 1993;64:1868-1873.

25. Loparic M, Wirz D, Daniels AU, et al. Micro- and nanomechanical analysis of articular cartilage by indentation-type atomic force microscopy: validation with a gel-microfiber composite. Biophys J. 98:2731-2740.

26. Oliver WC, Pharr G. An improved technique for determining hardness and elastic modulus using load and displacement sensing indentation experiments. J Mater Res. 1992;7:15641583.

27. Sundarraj N, Willson J. Monoclonal antibody to human basement membrane collagen type IV. Immunology. 1982; 47:133-140.

28. Libby RT, Champliaud MF, Claudepierre T, et al. Laminin expression in adult and developing retinae: evidence of two novel CNS laminins. J Neurosci. 2000;20:6517-6528.

29. Sado Y, Kagawa M, Kishiro Y, et al. Establishment by the rat lymph node method of epitope-defined monoclonal antibodies recognizing the six different alpha chains of human type IV collagen. Histochem Cell Biol. 1995;104:267-275.

30. Ljubimov AV, Burgeson RE, Butkowski RJ, et al. Extracellular matrix alterations in human corneas with bullous keratopathy, basement membrane abnormalities in human eyes with diabetic retinopathy, cleavage of human corneal type VI collagen alpha 3 chain by matrix metalloproteinase-2, proteolysis regulates exposure of the IIICS-1 adhesive sequence in plasma fibronectin. Invest Ophthalmol Vis Sci. 1996;37:9971007.

31. Binnig G, Quate CF, Gerber C. Atomic force microscope. Phys Rev Lett. 1986;56:930-933.
32. Last JA, Russell P, Nealey PF, Murphy CJ. The applications of atomic force microscopy to vision science. Invest Ophthalmol Vis Sci. 51:6083-6094.

33. Hu H, Candiello J, Zhang P, Ball SL, Cameron DA, Halfter W. Retinal ectopias and mechanically weakened basement membrane in a mouse model of muscle-eye-brain (MEB) disease congenital muscular dystrophy. Mol Vis. 1415;16: 1415-1428.

34. Bystrom B, Virtanen I, Rousselle P, Gullberg D, PedrosaDomellof F. Distribution of laminins in the developing human eye. Invest Ophthalmol Vis Sci. 2006;47:777-785.

35. Chen W, Mo W, Sun K, Huang X, Zhang YL, Song HY. Microplasmin degrades fibronectin and laminin at vitreoretinal interface and outer retina during enzymatic vitrectomy. Curr Eye Res. 2009;34:1057-1064.

36. Grazi E, Di Bona C. Protein osmotic pressure and cross-bridge attachment determine the stiffness of thin filaments in muscle ex vivo. $J$ Biochem. 2006;140:39-42.

37. Rowlands AS, George PA, Cooper-White JJ. Directing osteogenic and myogenic differentiation of MSCs: interplay of stiffness and adhesive ligand presentation. Am J Physiol Cell Physiol. 2008;295:C1037-1044

38. Brooks HL Jr. Macular hole surgery with and without internal limiting membrane peeling. Ophthalmology. 2000;107:19391948; discussion 1948-1939.

39. Foulquier S, Glacet-Bernard A, Sterkers M, Soubrane G, Coscas G. Study of internal limiting membrane peeling in stage- 3 and -4 idiopathic macular hole surgery [in French]. J Fr Ophtalmol. 2002;25:1026-1031.

40. Sheidow TG, Blinder KJ, Holekamp N, et al. Outcome results in macular hole surgery: an evaluation of internal limiting membrane peeling with and without indocyanine green. Ophthalmology. 2003;110:1697-1701.

41. Christensen UC, Kroyer K, Sander B, et al. Value of internal limiting membrane peeling in surgery for idiopathic macular hole stage 2 and 3: a randomised clinical trial. $\mathrm{Br} J$ Ophthalmol. 2009;93:1005-1015.

42. Trese MT, Chandler DB, Machemer R. Macular pucker. I. Prognostic criteria. Graefes Arch Clin Exp Opbthalmol. 1983; 221:12-15.

43. Henrich PB, Priglinger SG, Haritoglou C, et al. Quantification of contrast recognizability during brilliant blue $G$ - and indocyanine green-assisted chromovitrectomy. Invest Ophthalmol Vis Sci. 2011;52(7):4345-4349.

44. Gandorfer A, Scheler R, Haritoglou C, Schumann R, Nentwich M, Kampik A. pathology of the macular hole rim in flatmounted internal limiting membrane specimens. Retina. 2009;29:1097-1105.

45. Candiello J, Balasubramani M, Schreiber EM, et al. Biomechanical properties of native basement membranes. Febs J. 2007; 274:2897-2908.

46. Dalkara D, Kolstad KD, Caporale $\mathrm{N}$, et al. Inner limiting membrane barriers to AAV-mediated retinal transduction from the vitreous. Mol Ther. 2009;17:2096-2102. 